\title{
miR-21 attenuates contrast-induced renal cell apoptosis by targeting PDCD4
}

\author{
KUN WANG $^{1 *}$, WEI-JIE BEI ${ }^{1 *}$, YUAN-HUI LIU $^{1 *}$, HUA-LONG LI $^{1 *}$, SHI-QUN CHEN $^{1}$, \\ KAI-YANG LIN ${ }^{1}$, ZHI-LING ZHOU ${ }^{1}$, JI-YAN CHEN ${ }^{1,2}$, YONG LIU ${ }^{1,2}$ and NING TAN ${ }^{1,2}$ \\ ${ }^{1}$ Department of Cardiology, Guangdong Cardiovascular Institute, Guangdong Key Laboratory of \\ Coronary Disease, Guangdong General Hospital, Guangdong Academy of Medical Sciences, Guangzhou, Guangdong 510515; \\ ${ }^{2}$ School of Medicine, South China University of Technology, Guangzhou, Guangdong 510100, P.R. China
}

Received October 17, 2016; Accepted June 19, 2017

DOI: $10.3892 / \mathrm{mmr} .2017 .7426$

\begin{abstract}
Contrast medium (CM) is widely used in cardiac catheterization; however, it may induce acute kidney injury or renal failure, although the underlying mechanism remains to be elucidated. MicroRNA-21 (miR-21) is involved in renal disease and has been indicated to regulate cellular apoptosis and fibrosis, although its role in CM-induced renal cell injury is unknown. The present study examined the expression and potential targets of miR-21 in human renal proximal tubular epithelial (HK-2) cells following CM treatment. CM induced renal cell apoptosis and decreased miR-21 expression. The expression level of the apoptosis regulator protein, B-cell lymphoma 2 (Bcl-2) was upregulated, whereas that of the apoptosis regulator, Bcl-2-associated X protein (Bax) was downregulated upon transfection of miR-21 mimics; miR-21 overexpression additionally directly inhibited the expression of
\end{abstract}

Correspondence to: Dr Ning Tan or Dr Yong Liu, Department of Cardiology, Guangdong Cardiovascular Institute, Guangdong Key Laboratory of Coronary Disease, Guangdong General Hospital, Guangdong Academy of Medical Sciences, 106 Zhongshan Second Road, Guangzhou, Guangdong 510515, P.R. China

E-mail: tanning100@126.com

E-mail: liuyongmd@126.com

${ }^{*}$ Contributed equally

Abbreviations: miR-21, microRNA-21; PDCD4, programmed cell death protein 4; Bcl-2, B-cell lymphoma 2; Bax, Bcl-2-associated $\mathrm{X}$ protein; CM, contrast medium; CI-AKI, contrast-induced acute kidney injury; HK-2, human renal proximal tubular epithelial; HEK, human embryonic kidney; IRI, ischemia reperfusion injury; MUT, mutant type; WT, wild type; RT-qPCR, reverse transcription-quantitative polymerase chain reaction; siRNA, small interfering RNA; TUNEL, terminal deoxynucleotidyl transferase dUTP nick-end labeling

Key words: microRNA-21, programmed cell death protein 4, contrast medium, contrast-induced acute kidney injury, HK-2 cells programmed cell death protein 4 (PDCD4), as determined by a dual luciferase reporter assay, and PDCD4 silencing reduced the rate of HK-2 cell apoptosis. The results of the present study indicated that miR-21 protected renal cells against $\mathrm{CM}$-induced apoptosis by regulating PDCD4 expression.

\section{Introduction}

Contrast-induced acute kidney injury (CI-AKI) is the third most common cause of hospital-acquired renal failure, and is associated with increased cardiovascular and renal morbidity and mortality $(1,2)$. Although efforts have focused on the treatment and prevention of the development of renal dysfunction, its incidence is increasing $(3,4)$. Contrast medium (CM)-induced renal epithelial cell apoptosis is an important underlying cause of renal failure (5), although the mechanism remains unclear.

MicroRNAs (miRNAs/miRs) are a class of small, non-coding RNA molecules with a length of 18-25 nucleotides that serve an important role in normal biological functions, including cell growth, proliferation, differentiation and apoptosis, via post-transcriptional regulation of gene expression (6). Recent evidence has implicated miRNAs in ischemia-reperfusion injury (IRI), in addition to cisplatin-/cyclosporine-induced AKI (7-10). Specifically, miR-21 was observed to regulate renal cell apoptosis and fibrosis (11), although these finding are controversial, with certain studies demonstrating miR-21 upregulation $(12,13)$ and others reporting a downregulation, or no change, in expression levels $(14,15)$, depending on the tissue or disease model.

Programmed cell death protein 4 (PDCD4) is expressed in proliferating cells where it is known to suppress tumorigenesis and induce apoptosis, and is negatively regulated by miR-21 in various types of cancer (16). PDCD4 has been demonstrated to be associated with IRI (12). However, the role of PDCD4 in CM-induced renal cell injury has yet to be fully elucidated. It may be hypothesized that PDCD4 may be involved in renal tubular epithelial cell apoptosis induced by CM exposure (5). In order to test this hypothesis, the present study examined miR-21 expression levels in human renal proximal tubular epithelial (HK-2) cells under CM treatment and in gain- and loss-offunction experiments, to determine the association between miR-21 and PDCD4 expression and renal cell apoptosis. The 
results of the present study indicated that miR-21 protected kidney cells against CM-induced apoptosis by directly targeting PDCD4.

\section{Materials and methods}

Cell culture. HK-2 and human embryonic kidney (HEK)-293T cells were provided by the Stem Cell Bank, Chinese Academy of Sciences (Shanghai, China), and were cultured in Dulbecco's modified Eagle's medium/nutrient mixture F-12 (Gibco ${ }^{\mathrm{TM}}$; Thermo Fisher Scientific, Inc., Waltham, MA, USA) supplemented with $10 \%$ fetal bovine serum (Hyclone; GE Healthcare Life Sciences, Logan, UT, USA) at $37^{\circ} \mathrm{C}$ and $5 \% \mathrm{CO}_{2}$, and were subcultured when they reached $80-85 \%$ confluence. The cells were treated with $150 \mathrm{mg}$ iodide (mgI)/ml Ultravist CM (370 mgI/ml; Bayer AG, Leverkusen, Germany) for $2 \mathrm{~h}$ at $37^{\circ} \mathrm{C}$.

Terminal deoxynucleotidyl transferase dUTP nick-end labeling (TUNEL) assay. Cells $\left(2 \times 10^{6}\right)$ were washed with PBS and fixed with $4 \%$ paraformaldehyde for $30 \mathrm{~min}$ at room temperature. Following three washes with PBS, cells were treated with $0.2 \%$ Triton X-100 for 5 min on ice, washed with PBS, and incubated with fluorescein isothiocyanate-labeled dUTP and terminal deoxynucleotidyl transferase for $1 \mathrm{~h}$ at $37^{\circ} \mathrm{C}$. Following a series of washes with PBS and nuclear staining with DAPI at room temperature away from light, cells were analyzed with an epifluorescence microscope in five different visual fields at x400 magnification.

Target gene prediction. The Targetscan (www.targetscan. org) and microRNA.org databases (www.microrna. org/microrna/home.do) were used to predict the potential target of miR-21.

Reverse transcription-quantitative polymerase chain reaction (RT-qPCR) analysis. Total RNA was extracted using TRIzol reagent (Invitrogen; Thermo Fisher Scientific, Inc.), according to the manufacturer's protocol. Subsequent to the addition of $100 \%$ chloroform followed by $100 \%$ isopropanol, washes with $75 \%$ ethanol, and a series of centrifugation steps, RNA concentration and quality were measured by spectrophotometry at 260 and $280 \mathrm{~nm}$. Isolated RNA was reverse-transcribed using M-MLV reverse transcriptase (Takara Bio, Inc., Otsu, Japan), and the cDNA was amplified using SYBR Premix Ex Taq (Takara Bio, Inc.) on a CFX96 Touch real-time PCR detection system (Bio-Rad Laboratories, Inc., Hercules, CA, USA), according to a standard protocol under the following conditions: $95^{\circ} \mathrm{C}$ for $5 \mathrm{~min}$, followed by 40 cycles of $95^{\circ} \mathrm{C}$ for $10 \mathrm{sec}, 60^{\circ} \mathrm{C}$ for $10 \mathrm{sec}$, and $72^{\circ} \mathrm{C}$ for $10 \mathrm{sec}$. U6 was used as an internal control for miRNAs. The primer for miRNA amplification was synthesized by Sangon Biotech Co., Ltd. (Shanghai, China). The comparative threshold cycle $\left(2^{-\Delta \Delta \mathrm{Cq}}\right)$ method was used to analyze relative changes in gene expression (17). The primers were as follows: miR-21, forward 5'-ACGGGTAGC TTATCAGACTGA-3' and reverse 5'-CAGTGCGTGTCG TGGAGT-3'; PDCD4, forward 5'-AACCCTGCAGAAAAT GCTGG-3' and reverse 5'-CCTTAGTCGCCTTTTTGC CTTG-3'; and U6, forward 5'-CTCGCTTCGGCAGCACA-3' and reverse 5'-AACGCTTCACGAATTTGCGT-3'.
Western blotting. Cells were lysed by incubation with trypsin-EDTA solution (Invitrogen; Thermo Fisher Scientific, Inc.) and protein was extracted with radioimmunoprecipitation assay buffer (Thermo Fisher Scientific, Inc.) containing protease inhibitor. Protein concentration was quantified using a BCA Protein Assay kit (Thermo Fisher Scientific, Inc.). Equal amounts of protein $(20 \mu \mathrm{g})$ were separated by SDS-PAGE on a $12 \%$ gel and electrophoretically transferred to a polyvinylidene difluoride membrane, which was incubated in blocking solution containing 5\% non-fat milk in a Tris-buffered saline/Tween 20 solution (TBST) for $60 \mathrm{~min}$ at room temperature. Blocking was followed by overnight incubation at $4{ }^{\circ} \mathrm{C}$ with rabbit primary antibodies against the following proteins: PDCD4 (cat. no. 9535; 1:1,000), the apoptosis regulators, B-cell lymphoma 2 (Bcl-2; cat. no. 4223; 1:1,000) and Bcl-2-associated X protein (Bax; cat. no. 5023; 1:500) (all Cell Signaling Technology, Inc., Danvers, MA, USA); and $\beta$-actin (cat. no. ab8227; 1:1,000; Abcam, Cambridge, UK). Following washing with TBST, bound antibodies were detected by incubation for $60 \mathrm{~min}$ at room temperature with horseradish peroxidase-labelled goat anti-rabbit immunoglobulin G (cat. no. 7074; 1:5,000; Cell Signaling Technology, Inc.). Enhanced chemiluminescence (Thermo Fisher Scientific, Inc.) was used to visualize protein bands on X-ray film. Protein expression levels were normalized to that of $\beta$-actin. Image J v1.48 U (National Institutes of Health, Bethesda, MD, USA) was used for densitometric analysis.

Transfection of oligonucleotides and small interfering (si) $R N A$. Cells were seeded at a density of $2 \times 10^{5}$ in 6 -well plates and grown to $60-70 \%$ confluence in DMEM-F12, and subsequently transfected with miR-21 mimic or miR-21 inhibitor, scrambled control miR-21, siRNA against PDCD4 or negative control siRNA (Shanghai GenePharma Co., Ltd., Shanghai, China) using Lipofectamine 2000 (Invitrogen; Thermo Fisher Scientific, Inc.), according to the manufacturer's protocol. The working concentrations were $100 \mathrm{nM}$. After $48 \mathrm{~h}$, cells were treated with $150 \mathrm{mgI} / \mathrm{ml}$ Ultravist CM for $2 \mathrm{~h}$ at $37^{\circ} \mathrm{C}$. The efficiency of each mimic or inhibitor was confirmed by RT-qPCR. Proteins were detected by western blotting. The fraction of apoptotic cells was determined with the TUNEL assay. The sequences were as follows: miR-21-mimics, 5'-UAGCUUAUCAGACUGAUGUUGA-3'; miR-21-inhibitors, 5'-UCAACAUCAGUCUGAUAAGCU A-3'; and siRNA-PDCD4, 5'-GCGGUUUGUAGAAGAAUG UTT-3'.

Dual luciferase reporter assay. HEK-293T (2x10 $)$ cells were seeded in 24 -well plates $24 \mathrm{~h}$ prior to transfection. Cells were co-transfected with wild-type (WT) or mutant (MUT) PDCD4 untranslated region (UTR) constructs (pGL3-PDCD4-3'UTR-WT and pGL3-PDCD4-3'UTR-MUT, respectively) or the empty control vector pGL3-promoter pRL-TK (Promega Corporation, Madison, WI, USA) or miR-21 mimic (Guangzhou Ribobio Co., Ltd, Guangzhou, China) using Lipofectamine 2000. Relative luciferase activity was measured $48 \mathrm{~h}$ after transfection on a GloMax luminometer (Promega Corporation) and normalized to the firefly/Renilla luciferase signal in HEK-293T cells. 
A
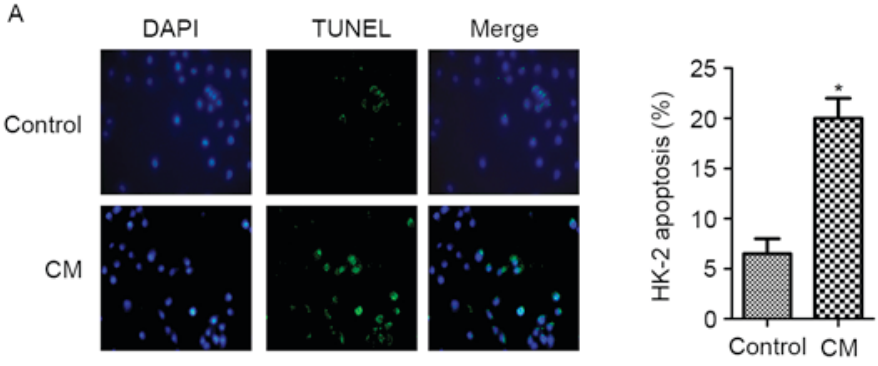

B

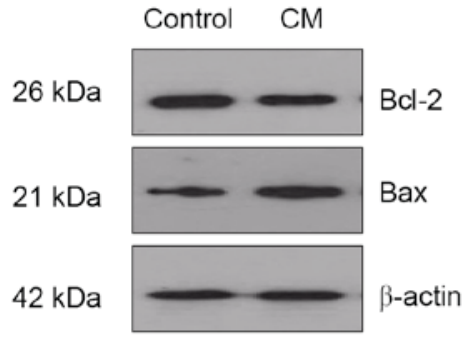

Control

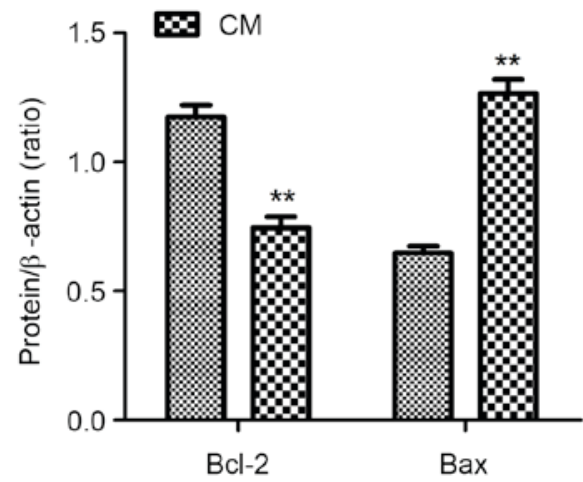

C

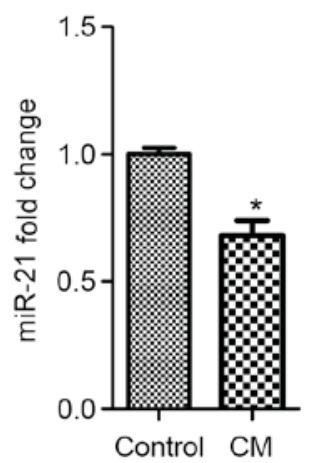

Figure 1. CM induces HK-2 cell apoptosis and inhibits miR-21 expression. (A) Apoptosis (green cells) was measured via the TUNEL assay. Magnification, x400. (B) Bcl-2 and Bax protein expression, as detected by western blotting. (C) MiR-21 expression, determined by the reverse transcription-quantitative polymerase chain reaction. Cells were treated with $150 \mathrm{mgI} / \mathrm{ml}$ Ultravist in the $\mathrm{CM}$ groups. ${ }^{*} \mathrm{P}<0.05$, ${ }^{* *} \mathrm{P}<0.01$ vs. control group ( $\mathrm{n}=3$ ). CM, contrast medium; miR, microRNA; TUNEL, terminal deoxynucleotidyl transferase dUTP nick-end labeling; Bcl-2, B-cell lymphoma 2; Bax, Bcl-2-associated X protein.

Statistical analysis. Data were described as mean \pm standard deviation. The determinations were performed at least in triplicate. An unpaired t-test and one way-analysis of variance (Bonferroni post hoc test for equal variances assumed; Tambane's T2 post hoc test for equal variances not assumed) were used to compare the groups using GraphPad Prism version 5.0 software (GraphPad Software, Inc., La Jolla, CA, USA) and SPSS software version 22.0 (IBM Corp., Armonk, $\mathrm{NY}$, USA). Two-tailed $\mathrm{P}<0.05$ was considered to indicate a statistically significant difference.

\section{Results}

$C M$ induces apoptosis and inhibits $m i R-21$ expression in $H K-2$ cells. HK-2 cells were treated with $150 \mathrm{mgI} / \mathrm{ml}$ Ultravist (370 $\mathrm{mgI} / \mathrm{ml}$ ) for $2 \mathrm{~h}$ and subsequently harvested for analysis. The rate of apoptosis was increased following CM treatment, as determined by the TUNEL assay (Fig. 1A). Consistent with this observation, the expression of the pro-apoptotic factor Bax was increased, whereas that of the anti-apoptotic factor $\mathrm{Bcl}-2$ was decreased under these conditions (Fig. 1B). Additionally, compared with untreated cells, the miR-21 level was downregulated by treatment with CM, as determined by RT-qPCR analysis (Fig. 1C), suggesting a negative association between miR-21 expression and HK-2 cell apoptosis in the presence of CM.

miR-21 overexpression inhibits CM-induced apoptosis in $H K-2$ cells. In order to investigate the effect of miR-21 on HK-2 cell apoptosis under CM treatment, cells were transfected with miR-21 mimic or inhibitor, or a negative control miRNA. The
miR-21 level was increased in cells transfected with mimic and reduced in inhibitor-treated cells, demonstrating a successful transfection (Fig. 2A). Western blot analysis revealed that Bax expression was downregulated, whereas that of Bcl-2 was upregulated, following transfection of the miR-21 mimic; the converse was observed in cells transfected with miR-21 inhibitor (Fig. 2B). Additionally, overexpression of miR-21 mimic decreased CM-induced apoptosis, whereas miR-21 inhibitor exerted the opposite effect, as determined by TUNEL assay (Fig. 2C). The results of the present study demonstrated that miR-21 may protect HK-2 cell against CM-induced apoptosis.

miR-21 inhibits HK-2 cell apoptosis by binding to the PDCD4 3' UTR. Target gene prediction indicated that PDCD4 may be a potential target of miR-21, since the PDCD4 3' UTR harbored a miR-21 binding site (Fig. 3A). In order to test the possibility of a miR-21 interaction with PDCD4, PDCD4 expression was evaluated in HK-2 cells transfected with miR-21 under CM treatment, using RT-qPCR analysis and western blotting. PDCD4 expression was upregulated in cells in the presence of CM (Fig. 3B and C); however, this effect was reversed by overexpression of miR-21 mimic, compared with cells transfected with negative control miR-21 mimic or those that were untransfected (Fig. 3D and E). Additionally, PDCD4 expression was increased in cells transfected with miR-21 inhibitor compared with the CM-only group, whereas the level was reduced upon transfection of miR-21 mimic (Fig. 3D and E), suggesting that miR-21 may attenuate apoptosis by inhibiting PDCD4 expression.

In order to confirm this hypothesis, constructs containing WT or MUT PDCD4 3' UTR were generated 

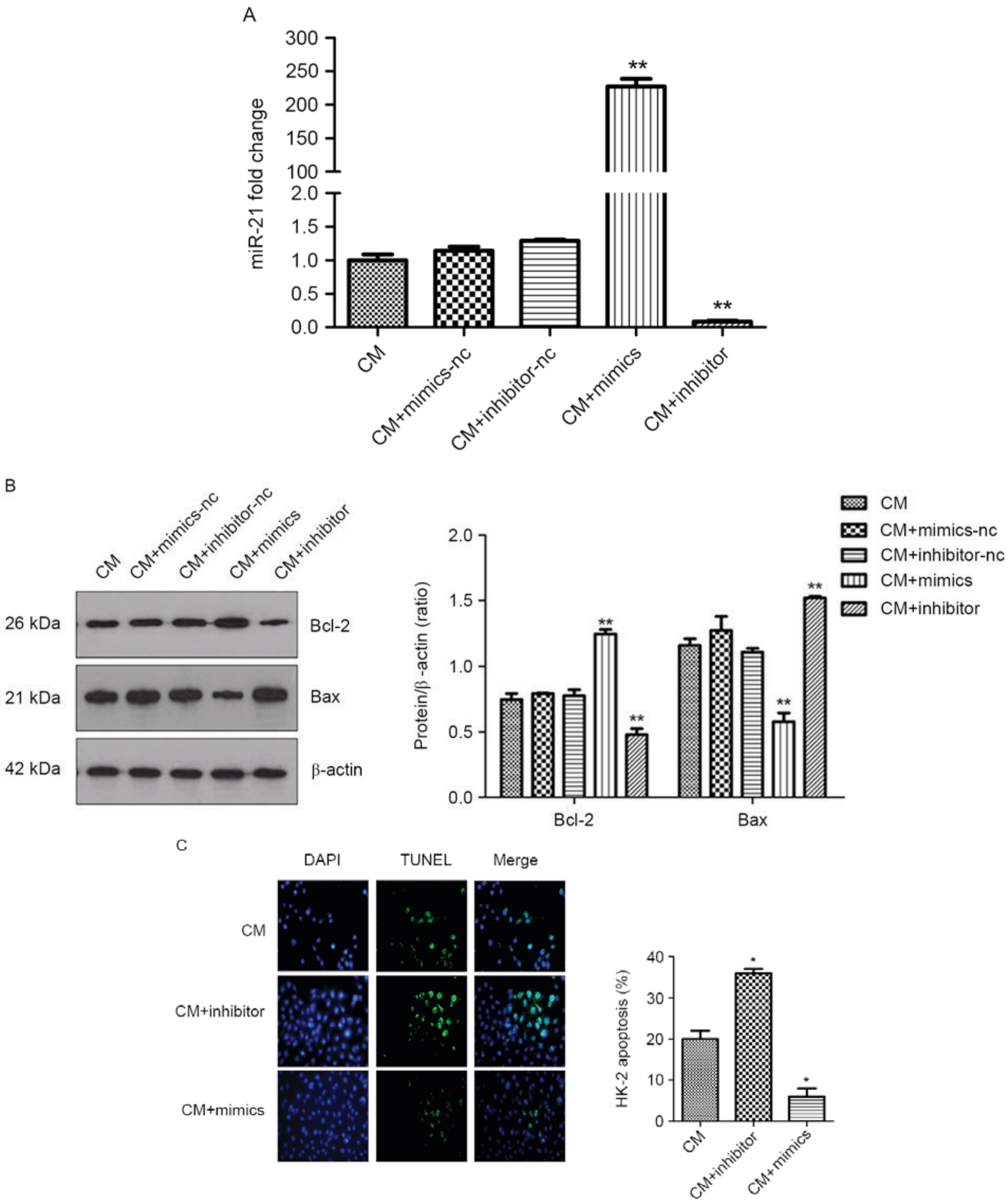

Figure 2. Effect of miR-21 on HK-2 cell apoptosis under CM treatment. (A) MiR-21 expression in cells transfected with miR-21 mimic, inhibitor, or negative control miR was detected using the reverse transcription-quantitative polymerase chain reaction. (B) Bcl-2 and Bax protein expression in cells transfected with miR-21 mimic, inhibitor or negative control miR was measured by western blotting. (C) Detection of apoptosis (green cells) with the TUNEL assay. Magnification, $x 400$. Cells were treated with $150 \mathrm{mgI} / \mathrm{ml}$ Ultravist in the $\mathrm{CM}$ groups. ${ }^{*} \mathrm{P}<0.05,{ }^{* *} \mathrm{P}<0.01$ vs. CM group (n=3). CM, contrast medium; miR, microRNA; HK-2, human renal proximal tubular epithelial; TUNEL, terminal deoxynucleotidyl transferase dUTP nick-end labeling; Bcl-2, B-cell lymphoma 2; Bax, Bcl-2-associated X protein.

(Fig. 4A). The luciferase activity in HEK-293T cells transfected with pGL3-PDCD4-3'UTR-WT was reduced by $\sim 50 \%$ relative to that in cells transfected with the MUT construct (Fig. 4B), suggesting that miR-21 may directly bind to the PDCD4 transcript, and thereby regulate its expression.
siRNA was used to knock down PDCD4 expression in HK-2 cells, and the effect of this on apoptosis was examined. PDCD4 mRNA and protein levels were decreased upon transfection of siRNA-PDCD4 (Fig. 4C and D). This decreased was accompanied by a downregulation of Bax and an upregulation of Bcl-2 compared with cells 
A

Human-miR-21mature sequence 3'...AGUUGUAGUCAGACUAUUCGAU...5

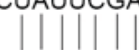

Position 242-249 of PDCD4 5'...AAGUGGAAUAUUCUAAUAAGCUA...3

B

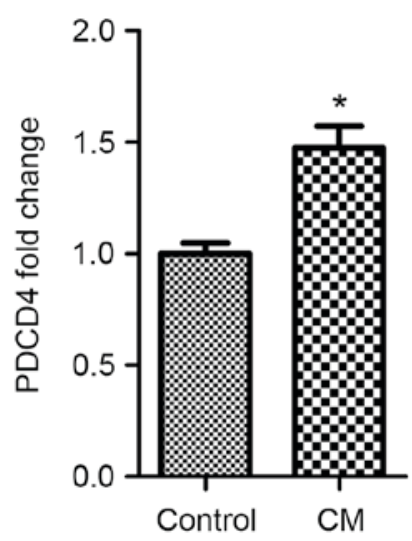

C

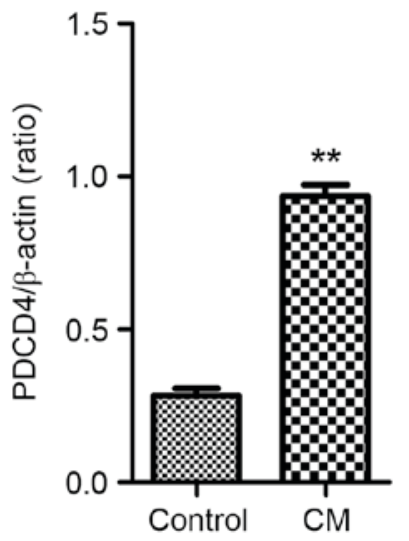

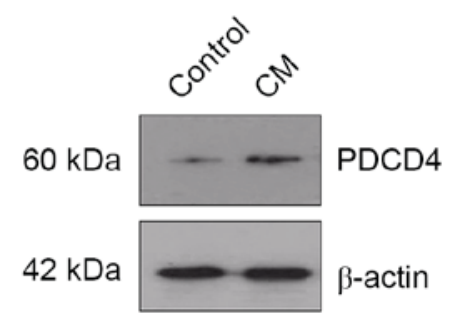

-actin

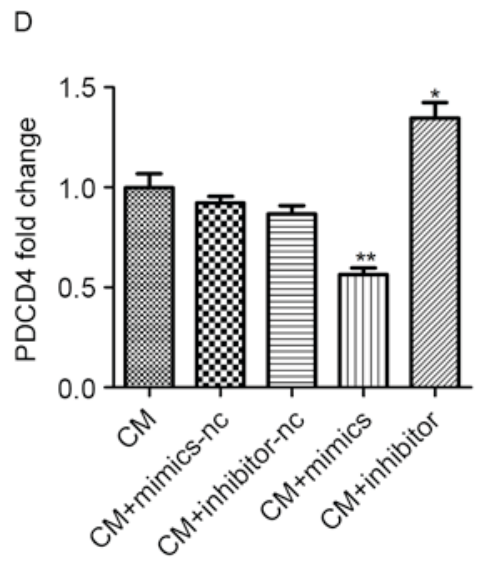

E
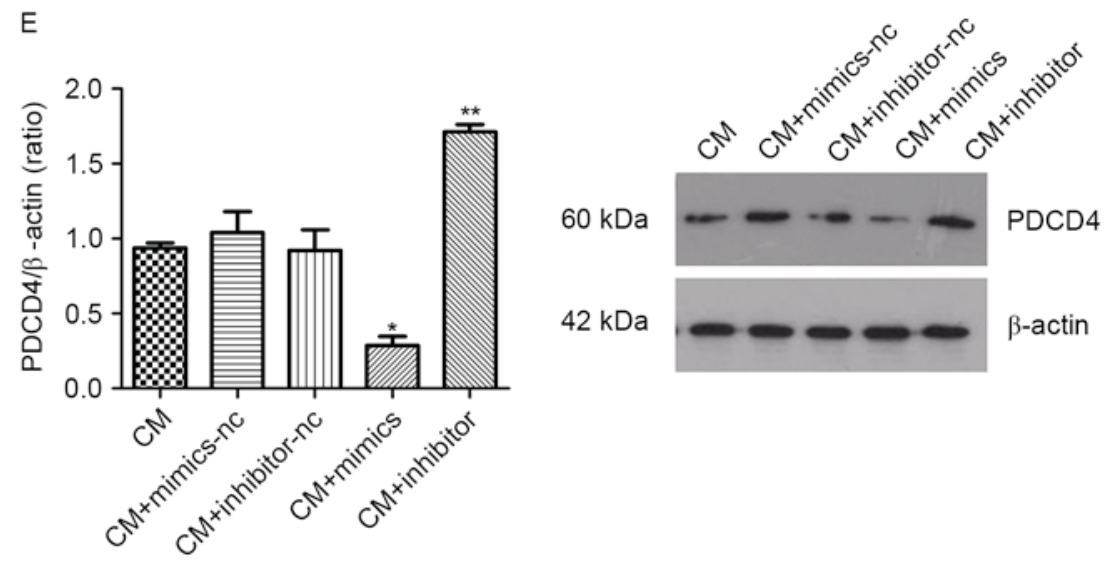

Figure 3. miR-21 inhibits PDCD4 expression. (A) Target gene analysis results, exhibiting the miR-21 binding sites in the 3' UTR of the human PDCD4 transcript. PDCD4 mRNA and protein levels in HK-2 cells treated with $150 \mathrm{mgI} / \mathrm{ml}$ Ultravist were determined by (B) reverse transcription-quantitative polymerase chain reaction and (C) western blotting, respectively. PDCD4 mRNA and protein expression in cells treated with $150 \mathrm{mgI} / \mathrm{ml}$ Ultravist following transfection with miR-21 mimic, inhibitor, or negative control miR was also measured by (D) reverse transcription-quantitative polymerase chain reaction and (E) western blotting, respectively. "P<0.05, " $\mathrm{P}<0.01$ vs. CM group $(\mathrm{n}=3)$. miR, microRNA; PDCD4, programmed cell death protein 4; UTR, untranslated region; HK-2, human renal proximal tubular epithelial; CM, contrast medium.

treated with CM only (Fig. 4D). The results of the present study demonstrated that miR-21 may protect renal cells from CM-induced apoptosis by suppressing PDCD4 expression.

\section{Discussion}

With the increasing use of $\mathrm{CM}$ in the diagnosis and treatment of coronary disease, CI-AKI has become the third leading cause of hospital-acquired AKI (18). Although the pathogenesis of CI-AKI is not well understood, apoptosis of renal epithelial and glomerular cells is hypothesized to be the underlying cause $(19,20)$. Bax and Bcl-2 are two important mediators of the mitochondrial apoptosis pathway (21); Bcl-2 prevents Bax activation and thereby inhibits apoptosis. It was previously reported that $\mathrm{Bcl}-2$ was downregulated in proximal renal tubular cells in AKI (22). As exhibited in the present study, Bax and Bcl-2 were increased and decreased, respectively, with a corresponding increase in the rate of apoptosis following $\mathrm{CM}$ treatment, consistent with previous reports of $\mathrm{CM}$-induced cell apoptosis.

miRNAs contribute to kidney homeostasis and disease (23). miR-21 has been implicated in apoptosis, fibrosis, inflammation and IRI $(24,25)$, and is considered to be a biomarker of AKI $(8,26)$. One study demonstrated that miR-21 was upregulated $24 \mathrm{~h}$ subsequent to IRI in an animal model (12); this was confirmed by other investigators who reported that miR-21 expression was increased in renal proximal tubular cells $24 \mathrm{~h}$ following hypoxia/reoxygenation or treatment with CM $(27,28)$. miR-21 levels have been observed to increase continuously in proliferating cells $(12,25)$, starting at $24 \mathrm{~h}$ post injury (29). Therefore, its expression did not increase within $24 \mathrm{~h}$ reperfusion subsequent to $20 \mathrm{~min}$ of ischemia, or within $8 \mathrm{~h}$ of CM administration $(13,30)$. It has been demonstrated that one-half of the amount of CM in the bloodstream may be eliminated quickly, in $\sim 2 \mathrm{~h}$ (31). In addition, the structure of renal cells exhibited moderate changes $2 \mathrm{~h}$ after $\mathrm{CM}$ administration (32). Therefore, 
A

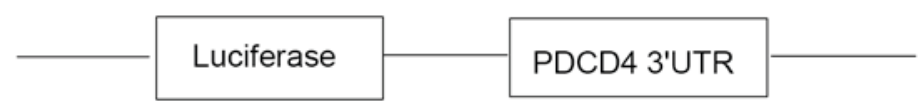

Human-miR-21mature sequence 3' ...AGUUGUAGUCAGACUAUUCGAU...5'

WT PDCD4 5' ...AAGUGGAAUAUUCUAAUAAGCUA...3' Mut PDCD4 5' ....AAGUGGAAUAUUCUACCCCAAGA....3'
B

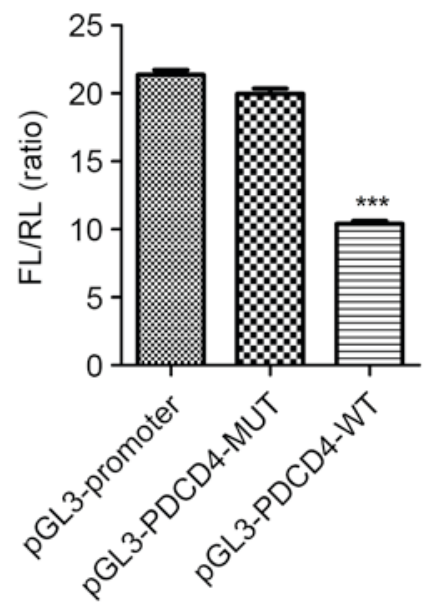

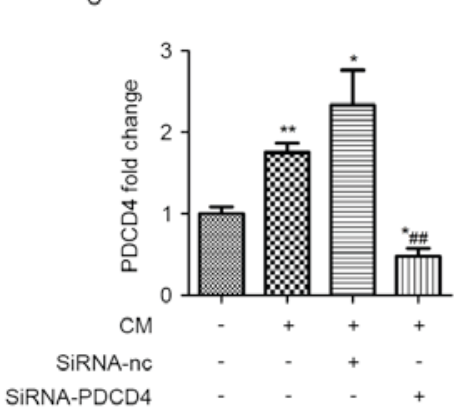

D

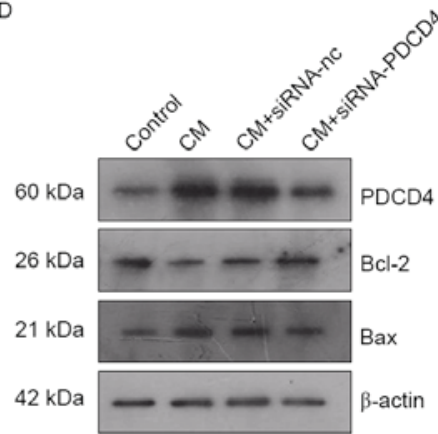

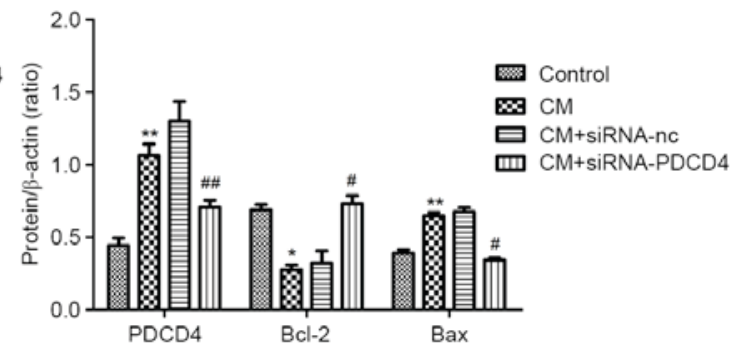

Figure 4. miR-21 inhibits renal cell apoptosis by directly targeting PDCD4 expression. (A) Plasmid with and without the miR-21 binding site in the 3' UTR sequence of PDCD4. The WT and MUT sequences were AUAAGCU and CCCCAAG, respectively (bold red letters). (B) Targeting of PDCD4 by miR-21, as determined with the dual-luciferase reporter assay. ${ }^{* * *} \mathrm{P}<0.001$ vs. MUT construct. (C) PDCD4 expression in HK-2 cells treated with siRNA against PDCD4. (D) PDCD4, Bcl-2, and Bax expression in HK-2 cells treated with siRNA against PDCD4, as determined by western blotting. ${ }^{*} \mathrm{P}<0.05$, ${ }^{* *} \mathrm{P}<0.01$ vs. control group; ${ }^{\#} \mathrm{P}<0.05$, ${ }^{\#} \mathrm{P}<0.01$ vs. CM group $(\mathrm{n}=3)$. miR, microRNA; PDCD4, programmed cell death protein 4; UTR, untranslated region; WT, wild-type; MUT, mutant; HK-2, HK-2, human renal proximal tubular epithelial; CM, contrast medium; siRNA, small interfering RNA; Bcl-2, B-cell lymphoma 2; Bax, Bcl-2-associated X protein; nc, negative control.

Neutrophil gelatinase-associated lipocalin, a novel biomarker of CI-AKI, has been demonstrated to begin to increase at $2 \mathrm{~h}$ post-CM exposure (33), although the level of serum creatinine did not significantly alter. In order to observe the effect of CM on renal cells, cells were incubated with CM for $2 \mathrm{~h}$ in the present study, and the expression of miR-21 was downregulated. Future studies are required to investigate alterations in miR-21 expression with respect to the degree of renal injury.

miR-21 was demonstrated to be renoprotective in animal and cellular models of IRI $(27,34)$. However, miR-21 has additionally been reported to exert deleterious effects in IRI, diabetic nephropathy and renal fibrosis (35-37). Physiological differences between models and variable times of ischemia may account for these conflicting observations. miR-21 was observed to regulate tumor cell proliferation, invasion, apoptosis and migration by targeting PDCD4 and Bcl-2 (38), while PDCD4 is thought to inhibit neoplastic transformation (39). As demonstrated in the present study, the levels of PDCD4 were reduced and enhanced upon transfection with a miR-21 mimic and inhibitor, respectively. In addition, the rate of apoptosis was decreased following PDCD4 knockdown. The results of the present study corroborated previous findings and demonstrated that PDCD4 may be negatively regulated by miR-21; additionally, these results suggested that the miR-21/PDCD4 pathway serves an important role in preventing CM-induced renal tubular cell apoptosis. To the best of our knowledge, the present study is the first to analyze the mechanism of CM-induced renal cell apoptosis from a genetic perspective. Whether the miR-21/PDCD4 pathway exhibits this function in vivo will be investigated in future studies.

In conclusion, the present study demonstrated that miR-21 protected renal cells against $\mathrm{CM}$-induced apoptosis by directly regulating PDCD4. The results of the present study may provide a basis for the development of therapeutic strategies to treat CI-AKI.

\section{Acknowledgements}

The present study was supported by the National Science Foundation for Young Scientists of China (grant no. 81500520), the National Natural Science Foundation of China (grant no. 81270286), and the Progress in Science and Technology Project of Guangdong Province (grant nos. 2013b031800025 and 2016b020215130). 


\section{References}

1. Nash K, Hafeez A and Hou S: Hospital-acquired renal insufficiency. Am J Kidney Dis 39: 930-936, 2002.

2. Tsai TT, Patel UD, Chang TI, Kennedy KF, Masoudi FA, Matheny ME, Kosiborod M, Amin AP, Messenger JC, Rumsfeld JS and Spertus JA: Contemporary incidence, predictors, and outcomes of acute kidney injury in patients undergoing percutaneous coronary interventions: Insights from the NCDR Cath-PCI registry. JACC Cardiovasc Interv 7: 1-9, 2014.

3. Hernandez GT and Nasri H: World Kidney Day 2014: Increasing awareness of chronic kidney disease and aging. J Renal Inj Prev 3: 3-4, 2014.

4. Nicola R, Shaqdan KW, Aran K, Mansouri M, Singh A and Abujudeh HH: Contrast-induced nephropathy: Identifying the risks, choosing the right agent and reviewing effective prevention and management methods. Curr Probl Diagn Radiol 44: 501-504, 2015.

5. Perrin T, Descombes E and Cook S: Contrast-induced nephropathy in invasive cardiology. Swiss Med Wkly 142: w13608, 2012.

6. Schickel R, Boyerinas B, Park S and Peter ME: MicroRNAs: Key players in the immune system, differentiation, tumorigenesis and cell death. Oncogene 27: 5959-5974, 2008.

7. Schena FP, Serino G and Sallustio F: MicroRNAs in kidney diseases: New promising biomarkers for diagnosis and monitoring. Nephrol Dial Transpl 29: 755-763, 2014.

8. Du J, Cao X, Zou L, Chen Y, Guo J, Chen Z, Hu S and Zheng Z: MicroRNA-21 and risk of severe acute kidney injury and poor outcomes after adult cardiac surgery. PLoS One 8: e63390, 2013

9. Shapiro MD, Bagley J, Latz J, Godwin JG, Ge X, Tullius SG and Iacomini J: MicroRNA expression data reveals a signature of kidney damage following ischemia reperfusion injury. PLoS One 6: e23011, 2011

10. Pavkovic M, Riefke B and Ellinger-Ziegelbauer H: Urinary microRNA profiling for identification of biomarkers after cisplatin-induced kidney injury. Toxicology 324: 147-157, 2014

11. Li YF, Jing Y, Hao J, Frankfort NC, Zhou X, Shen B, Liu X, Wang L and Li R: MicroRNA-21 in the pathogenesis of acute kidney injury. Protein Cell 4: 813-819, 2013.

12. Godwin JG, Ge X, Stephan K, Jurisch A, Tullius SG and Iacomini J: Identification of a microRNA signature of renal ischemia reperfusion injury. Proc Natl Acad Sci USA 107: 14339-14344, 2010.

13. Kaucsár T, Révész C, Godó M, Krenács T, Albert M, Szalay CI, Rosivall L, Benyó Z, Bátkai S, Thum T, et al: Activation of the miR-17 Family and miR-21 During murine kidney ischemia-reperfusion injury. Nucleic Acid Ther 23: 344-354, 2013.

14. Saikumar J, Hoffmann D, Kim TM, Gonzalez VR, Zhang Q, Goering PL, Brown RP, Bijol V, Park PJ, Waikar SS and Vaidya VS: Expression, circulation, and excretion profile of MicroRNA-21, -155 and -18a following acute kidney injury. Toxicol Sci 129: 256-267, 2012.

15. Kanki M, Moriguchi A, Sasaki D, Mitori H, Yamada A, Unami A and Miyamae Y: Identification of urinary miRNA biomarkers for detecting cisplatin-induced proximal tubular injury in rats. Toxicology 324: 158-168, 2014

16. Allgayer $\mathrm{H}: \mathrm{Pdcd} 4$, a colon cancer prognostic that is regulated by a microRNA. Crit Rev Oncol Hematol 73: 185-191, 2010.

17. Livak KJ and Schmittgen TD: Analysis of relative gene expression data using real-time quantitative PCR and the 2(-Delta Delta C(T)) Method. Methods 25: 402-408, 2001.

18. Aurelio A and Durante A: Contrast-induced nephropathy in percutaneous coronary interventions: Pathogenesis, risk factors, outcome, prevention and treatment. Cardiology 128: 62-72, 2014.

19. Zhang J, Duarte CG and Ellis S: Contrast medium- and mannitol-induced apoptosis in heart and kidney of SHR rats. Toxicol Pathol 27: 427-435, 1999.

20. Romano G, Briguori C, Quintavalle C, Zanca C, Rivera NV, Colombo A and Condorelli G: Contrast agents and renal cell apoptosis. Eur Heart J 29: 2569-2576, 2008.
21. Borkan SC: The Role of BCL-2 Family Members in Acute Kidney Injury. Semin Nephrol 36: 237-250, 2016.

22. Havasi A and Borkan SC: Apoptosis and acute kidney injury. Kidney Int 80: 29-40, 2011

23. Chandrasekaran K, Karolina DS, Sepramaniam S, Armugam A, Wintour EM, Bertram JF and Jeyaseelan K: Role of microRNAs in kidney homeostasis and disease. Kidney Int 81: 617-627, 2012.

24. Lai JY, Luo J, O'Connor C, Jing X, Nair V, Ju W, Randolph A, Ben-Dov IZ, Matar RN, Briskin D, et al: MicroRNA-21 in Glomerular Injury. J Am Soc Nephrol 26: 805-816, 2015.

25. Xu X, Kriegel AJ, Liu Y, Usa K, Mladinov D, Liu H, Fang Y, Ding X and Liang M: Delayed ischemic preconditioning contributes to renal protection by upregulation of miR-21. Kidney Int 82: $1167-1175,2012$

26. Ramachandran K, Saikumar J, Bijol V, Koyner JL, Qian J, Betensky RA, Waikar SS and Vaidya VS: Human miRNome Profiling Identifies MicroRNAs Differentially Present in the Urine after Kidney Injury. Clin Chem 59: 1742-1752, 2013.

27. Zhang $\mathrm{W}$ and Shu L: Upregulation of miR-21 by Ghrelin ameliorates ischemia/reperfusion-induced acute kidney injury by inhibiting inflammation and cell apoptosis. DNA Cell Biol 35: 417-425, 2016.

28. Gutiérrez-Escolano A, Santacruz-Vázquez E and Gómez-Pérez F: Dysregulated microRNAs involved in contrast-induced acute kidney injury in rat and human. Ren Fail 37: 1498-1506, 2015.

29. Humphreys BD, Czerniak S, DiRocco DP, Hasnain W, Cheema R and Bonventre JV: Repair of injured proximal tubule does not involve specialized progenitors. Proc Natl Acad Sci USA 108: 9226-9231, 2011

30. Sun SQ, Zhang T, Ding D, Zhang WF, Wang XL, Sun Z, Hu LH, Qin SY, Shen LH and He B: Circulating MicroRNA-188, -30a, and $-30 \mathrm{e}$ as early biomarkers for contrast-induced acute kidney injury. J Am Heart Assoc 5: pii: e004138, 2016.

31. Geenen RW, Kingma HJ and van der Molen AJ: Contrast-induced nephropathy: pharmacology, pathophysiology and prevention. Insights Imaging 4: 811-820, 2013.

32. Tervahartiala P, Kivisaari L, Kivisaari R, Vehmas $T$ and Virtanen I: Structural changes in the renal proximal tubular cells induced by iodinated contrast media. Nephron 76: 96-102, 1997.

33. Hirsch R, Dent C, Pfriem H, Allen J, Beekman RH III, Ma Q, Dastrala S, Bennett M, Mitsnefes M and Devarajan P: NGAL is an early predictive biomarker of contrast-induced nephropathy in children. Pediatr Nephrol 22: 2089-2095, 2007.

34. Jia P, Teng J, Zou J, Fang Y, Zhang X, Bosnjak ZJ, Liang M and Ding X: miR-21 contributes to xenon-conferred amelioration of renal ischemia-reperfusion injury in mice. Anesthesiology 119: 621-630, 2013

35. Liu X, Hong Q, Wang Z, Yu Y, Zou X and Xu L: MiR-21 inhibits autophagy by targeting Rablla in renal ischemia/reperfusion. Exp Cell Res 338: 64-69, 2015.

36. Zhong X, Chung AC, Chen HY, Dong Y, Meng XM, Li R, Yang W, Hou FF and Lan HY: miR-21 is a key therapeutic target for renal injury in a mouse model of type 2 diabetes. Diabetologia 56 : 663-674, 2013

37. Chau BN, Xin C, Hartner J, Ren S, Castano AP, Linn G, Li J, Tran PT, Kaimal V, Huang X, et al: MicroRNA-21 promotes fibrosis of the kidney by silencing metabolic pathways. Sci Transl Med 4: 118ra-121ra, 2012.

38. Xu LF, Wu ZP, Chen Y, Zhu QS, Hamidi S and Navab R: MicroRNA-21 (miR-21) regulates cellular proliferation, invasion, migration, and apoptosis by targeting PTEN, RECK and Bcl-2 in lung squamous carcinoma, Gejiu City, China. PLoS One 9: e103698, 2014

39. Asangani IA, Rasheed SA, Nikolova DA, Leupold JH, Colburn NH, Post S and Allgayer H: MicroRNA-21 (miR-21) post-transcriptionally downregulates tumor suppressor Pdcd4 and stimulates invasion, intravasation and metastasis in colorectal cancer. Oncogene 27: 2128-2136, 2007. 